\section{Compression after vein harvesting for coronary bypass}

\author{
Oscar Maleti \\ Department of Vascular Surgery, \\ Hesperia Hospital, Modena, Italy
}

\section{Introduction}

Using the saphenous vein for venous or coronary bypass is like inviting Cinderella to the Royal Ball because, instead of being ablated, it is used as a tool for revascularization.

The saphenous wall is particularly useful in this role because it hardly dilate. The low incidence of post-arterialization dilatation is indirect evidence that the wall dilatation that occurs in varicose veins is due to parietal stress, which is correlated with turbulence rather than with increased pressure.

Though the saphenous vein is the best material for bypass interventions, on the other side it is difficult to harvest.

There are different kinds of harvesting, depending on the type of skin incision, long or short, or performed in endoscopy.

\section{Saphenous vein harvesting}

Saphenous harvesting can be performed by means of open or endoscopic surgery. The open technique involves a long incision or multiple incisions. The long incision (Figure 1) improves the graft visibility, is more rapid and improves long-term graft patency, but it is associated with increased skin healing difficulty. The endoscopic surgery improves with multiple incisions (Figure 2), but this technique requires a longer operation and the reduced graft visibility is correlated with reduced long-term graft patency. The leg skin healing is faster compared to the first procedure. Considering that this technique is slower and more expensive, it is not currently applied.

The site of incision is important: at thigh level healing is usually better than at leg level; however, the discrepancy in caliber at thigh level, forces us to choose leg level.

The incidence of global complications is higher in women and the total complication rate is extremely variable, from $1 \%$ to $24 \%$.

Possible complications correlated to saphenous vein harvesting are shown in Table 1.

Certain patient characteristics may increase the harvesting complications rate; such features are age, dystrophic lesions, and associated vascular diseases (arterial, venous and lymphatic disease). Age and dystrophic lesions increase the time of incision healing, while obesity increases edema and postoperative cellulitis.

An associated arterial disease does not present particular problems in patients with an ankle-brachial pressure index (ABPI) 0.5-1 but in cases of ABPI less then 0.5 , problems of incision healing can occur correlated to suture, wound care and compression (Figure 3 ).

An associated venous disease presents two possible problems: the absence of a donor segment or the unsuitability of the donor segment in patients with chronic venous insufficiency (CVI). In the first case an alternative donor site is sought, while in CVI the patient will require adequate postoperative compression.

Harvesting in patients with associated lymphatic disease is particularly dangerous due to the increased risk of lymphedema worsening.

\section{Diagnostic approach}

Any candidate for saphenous harvesting should be submitted to preliminary ultrasound evaluation; this is able to detect arterial disease and CVI, and can also evaluate ABPI, femoral lesions (useful in catheterisation) and saphenous mapping (useful in guiding the incision).

In particular circumstances, supplementary diagnosis such as arteriography or venography may be necessary.

\section{Procedural strategy to reduce harvesting complications}

There is no correlation between postoperative edema and diabetes, the use of diuretics, low-sodium diet, poor-skin healing and incision length; however, the postoperative edema and the healing-reduction rate are correlated with the surgical technique employed and with postoperative treatment.

Regarding the surgical technique, it is preferable to avoid any border skin damage and to perform a single layer suture (Figure 4).

Compression therapy is crucial for reducing postoperative complications and should take account of the principal features of these patients: coagulation is normal; the patient remains in the supine position for two days; in the sitting position on the third postoperative day; in the standing position on the fourth postoperative day.

An anti-thrombotic elastic stocking is well tolerated by the patient, but this device exerts low compression at the saphenous-vein harvesting site. The use of short-stretch bandaging (concentric compression) has the advan-
Correspondence: 0scar Maleti, Department of Vascular Surgery, Hesperia Hospital, via Arquà, 80/A, 41125 Modena, Italy.

E-mail: oscarmaleti@gmail.com

This work is licensed under a Creative Commons Attribution 4.0 License (by-nc 4.0).

(C) Copyright O. Maleti, 2016

Licensee PAGEPress, Italy

Veins and Lymphatics 2016; 5:5989

doi:10.4081/vl.2016.5989

tage of being modifiable but it must be performed by experienced personnel and must be renewed daily. The application of eccentric compression at the harvesting site has the advantage of exerting specific compression where needed, reducing the risk of hematoma (Figure 5), and it is also applicable in patients with arterial disease. This kind of compression must also be performed by experienced personnel, and it is time consuming. Crucial points to remember in postoperative compression are: bandage pressure within the limits of patient pathology; protect the skin; protect areas at risk of decubitus; remove and check bandage on second postoperative day. After discharge, the elastic stocking should be maintained for four weeks, as the third and fourth weeks are a crucial period in skin healing.

\section{Conclusions}

Although it has been always considered of secondary importance and thus neglected, proper harvesting at the saphenous vein site plays a crucial role in candidates for coronary bypass operation. Correct management of the harvesting site and effective postoperative control of hemostasis and edema by means of compression therapy considerably reduces the length of the hospital stay and related costs.

Table 1. Possible complication correlated to saphenous vein harvesting.

\begin{tabular}{l} 
- Edema \\
- Early \\
\hline - Late (4 weeks) \\
- Lymphedema \\
- Hematoma \\
- Erythema \\
- Infection \\
- Bad incision healing \\
- Saphenous vein neuralgia \\
- Stump deep venous thrombosis \\
- Cellulitis
\end{tabular}




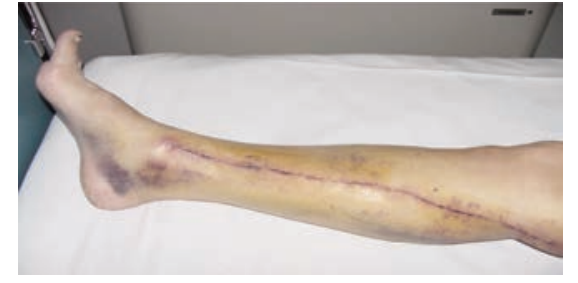

Figure 1. Open technique: long incision.

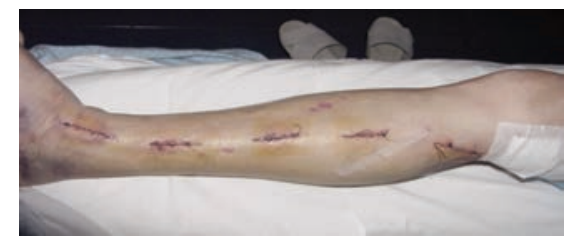

Figure 2. Open technique: multiple incisions.

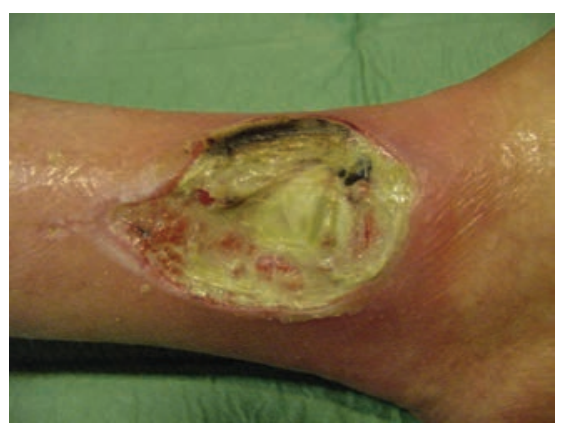

Figure 3. Bad healing in patient with arteriopathy.

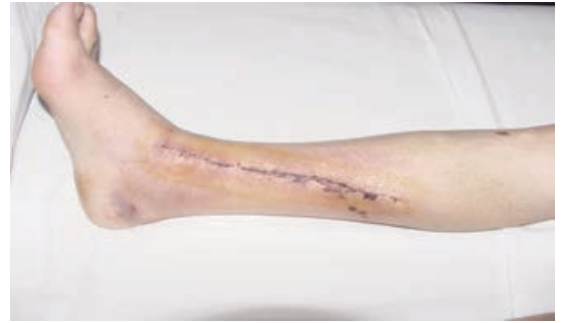

Figure 4. Bad healing of the incision due to margins damage.

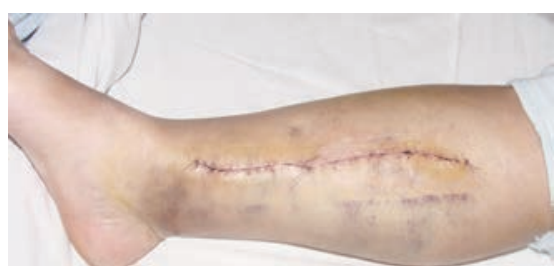

Figure 5. Hematoma due to wrong compression. 